\title{
In situ genotyping individual DNA molecules by target-primed rolling-circle amplification of padlock probes
}

\author{
Chatarina Larsson ${ }^{1}$, Jørn Koch ${ }^{2}$, Anders Nygren ${ }^{1}$, George Janssen ${ }^{3}$, Anton K Raap ${ }^{3}$, Ulf Landegren ${ }^{1}$ \\ \& Mats Nilsson ${ }^{1}$
}

Methods are needed to study single molecules to reveal variability, interactions and mechanisms that may go undetected at the level of populations of molecules. We describe here an integrated series of reaction steps that allow individual nucleic acid molecules to be detected with excellent specificity. Oligonucleotide probes are circularized after hybridization to target sequences that have been prepared so that localized amplification reactions can be initiated from the target molecules. The process results in strong, discrete detection signals anchored to the target molecules. We use the method to observe the distribution, within and among human cells, of individual normal and mutant mitochondrial genomes that differ at a single nucleotide position.

By analyzing biological processes at the ultimate level of single molecules, and with sufficient precision to distinguish even closely similar variants, it will be possible to study the inter- or subcellular context of specific DNA or RNA sequences as well as to analyze their location among extensive, arrayed sets of biological samples. Unlike simple hybridization probes, PCR provides adequate specificity to detect unique target sequences in the context of whole genomes, but the technique is poorly suited for precisely localized detection reactions. Oligonucleotide probes with one targetcomplementary sequence at each end-padlock probes—encircle specific target molecules as a consequence of templated DNA ligation. These probes have detection specificity similar to that obtained with $\mathrm{PCR}^{1-3}$, but unlike in PCR, the reaction products can remain bound to their target sequences, even withstanding denaturing washes ${ }^{4}$. Unfortunately, detection signals from specifically circularized padlock probes have proven insufficient to reveal single-copy gene sequences owing to background noise from nonspecifically bound probes, although repeated centromeric sequences have been evaluated with sufficient precision to investigate the distribution of single-nucleotide variants in situ ${ }^{5,6}$.

Linear oligonucleotide probes hybridized in situ have been used as primers for site-specific DNA synthesis, allowing labeled nucleotides to be incorporated in the so-called PRINS reaction ${ }^{7}$. This process enables localized allele-specific detection of repeated sequences in the genome $\mathrm{e}^{8-11}$, but this method, too, falls short of detecting singlecopy genes from single primers, as would be needed for genotyping in situ. More recently, linear probes hybridized in situ have been extended not by replicating the target sequence, but by using separately added small circular DNA strands as templates for a localized rolling-circle amplification (RCA) ${ }^{12,13}$. Although this results in clearly detectable signals from individual probes, the specificity of the reaction may be insufficient for robust detection of unique single-copy sequences in situ because of difficulties in distinguishing specifically bound from nonspecifically bound probes.

If, instead, the reaction is set up so that the RCA template can form only in a target-specific circularization reaction in situ, nonspecific signals should be greatly diminished; and promising results have been reported with this approach ${ }^{14,15}$. Regrettably, replication of circular probes that remain wound around their target strands seems to be inhibited, probably as a consequence of molecular crowding ${ }^{16}$, although there is some controversy in the literature about this topological inhibition ${ }^{17}$. In an interesting approach, target sequences were enzymatically rendered single stranded having free $5^{\prime}$ ends near the binding sites for padlock probes ${ }^{15}$. Upon addition of a primer the probes can be replicated in situ by RCA, but only if they escape the topological block against replication by detaching from the target molecules. In the process, amplification products risk drifting away, however, potentially further reducing the efficiency of localized detection.

We now present a procedure that integrates highly specific and sensitive recognition of target nucleic acid sequences using padlock probes with efficient local signal amplification, thereby permitting detection of single-nucleotide variation of individual DNA molecules in situ. Padlock probes, circularized in a target-dependent manner, serve as templates for uninhibited rolling circle reactions primed from the actual target sequences. In this way, the anchored target molecules become extended by hundreds of copies of the reacted probes, readily available for detection using standard

${ }^{1}$ Department of Genetics and Pathology, Rudbeck Laboratory, Uppsala University, Se-75185 Uppsala, Sweden. ${ }^{2}$ Institute of Pathology, Aarhus Sygehus, Norrebrogade 44, DK-8000 Aarhus C, Denmark. ${ }^{3}$ Department of Molecular Cell Biology, Leiden University Medical Center, Wassenaarseweg 72, 2333A. Correspondence should be addressed to M.N. (Mats.nilsson@genpat.uu.se).

PUBLISHED ONLINE 18 NOVEMBER 2004; DOI:10.1038/NMETH723 


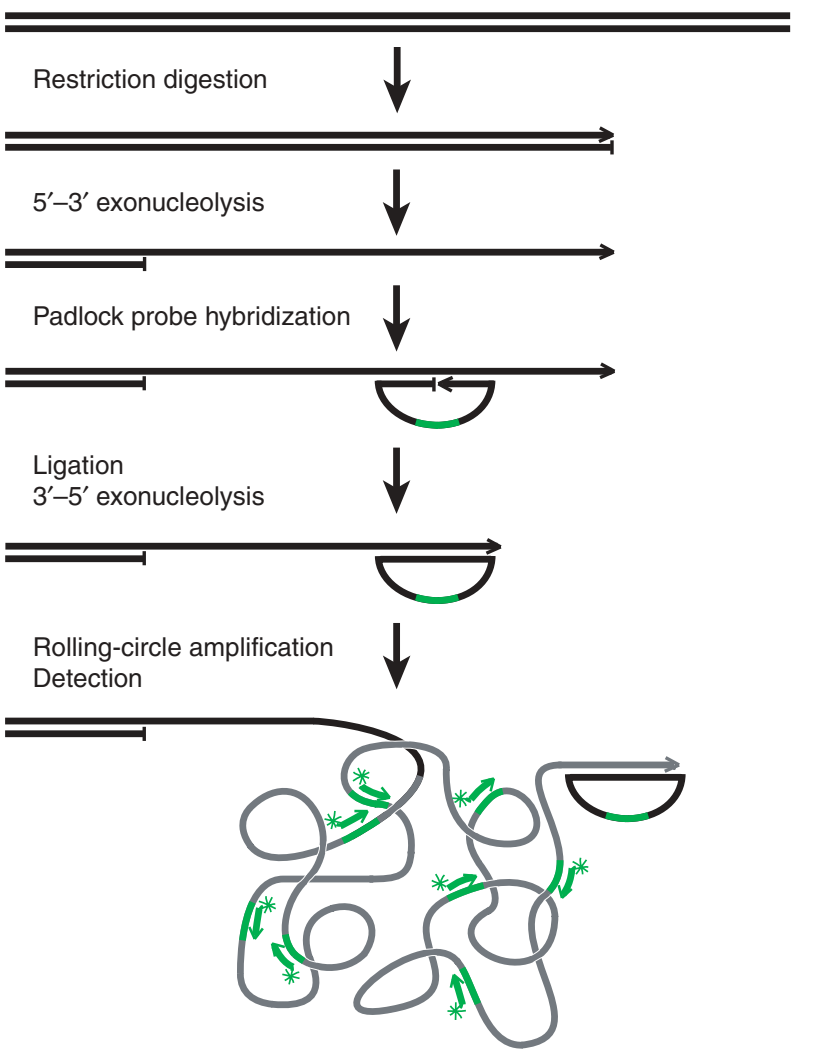

Figure 1 | Schematic representation of target-primed rolling-circle amplification of circularized padlock probes. The target DNA is restriction digested $3^{\prime}$ of the target sequence and irreversibly made single stranded by strand-specific $5^{\prime}-3^{\prime}$ exonucleolysis. Padlock probes are hybridized to their target sequences and the probe ends are joined through ligation, locking the probe onto the target molecule. After ligation, the RCA is initiated by the Ф29 DNA polymerase by turning the target molecule into a primer through $3^{\prime}-5^{\prime}$ exonucleolysis of any $3^{\prime}$ end protruding beyond the padlock probe hybridization site. The padlock probe then serves as the template for DNA synthesis. The RCA product (gray) is detected through hybridization of fluorescence-labeled oligonucleotides to tag sequences (green) specific for the padlock probe. Arrowheads indicate $3^{\prime}$ ends and bars $5^{\prime}$ ends.

hybridization probes. We compared our method to other approaches, and used it to observe the distribution of normal mitochondrial genomes and ones harboring a single-nucleotide variant, characteristic of the MELAS syndrome, in homo- and heteroplasmic cell lines and in histological tissue sections.

\section{RESULTS}

\section{Method Design}

Normal and mutant mitochondria often coexist in the cells of patients with mitochondrial disease, a phenomenon termed heteroplasmy, and the proportions can shift markedly from mother to offspring or between somatic cell generations ${ }^{18}$. Here we have designed padlock probes to genotype the mitochondrial A3243G mutation in situ. This mutation is observed in the severe but rare MELAS syndrome (mitochondrial myopathy, encephalopathy, lactic acidosis and stroke-like episodes, OMIM 540000) ${ }^{19}$ and in the more common MIDD syndrome (maternally inherited diabetes and deafness, OMIM 520000), which may account for as much as $1 \%$ of cases of diabetes ${ }^{20}$.

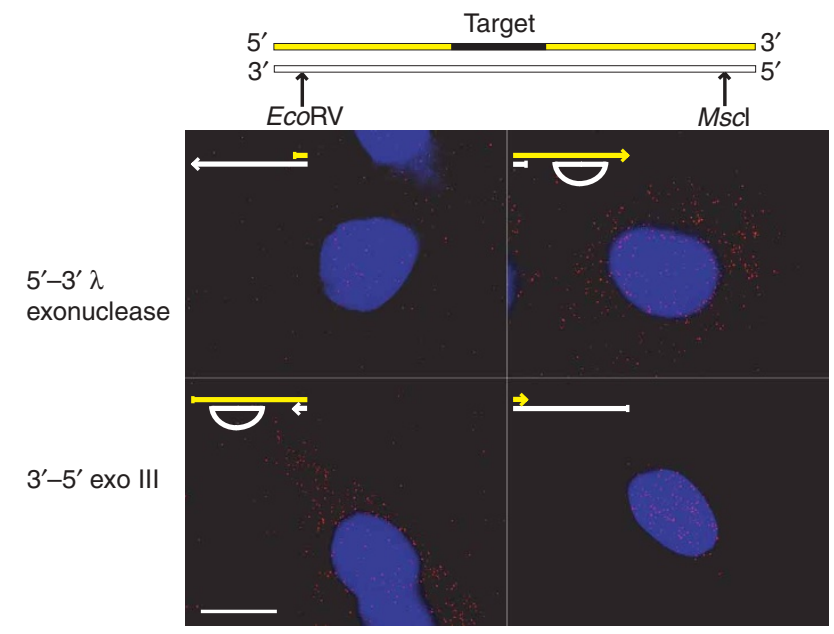

Figure 2 | Assessment of enzymatic target preparation of mtDNA in cultured cells. Nuclei were stained with DAPI (blue) and digoxigenin-labeled padlock probes hybridized and ligated to the target sequence were visualized by immunohistochemistry (red). A map of the target and restriction sites is shown above the images, and insets show the expected configurations of the target fragments. The sense strand is shown in yellow and the antisense strand in white. Arrowheads in insets indicate $3^{\prime}$ ends and bars $5^{\prime}$ ends. The cells shown in the left column were restriction digested $5^{\prime}$ of the target sequence using EcoRV, and the cells shown in the right column were digested $3^{\prime}$ of the target sequence using MscI. The cells shown in the top row were treated with a $5^{\prime}-3^{\prime}$ exonuclease ( $\lambda$ exonuclease), and the cells shown in the bottom row were treated with a $3^{\prime}-5^{\prime}$ exonuclease (exonuclease III) after restriction digestion. Scale bar, $20 \mu \mathrm{m}$.

By amplifying circularized padlock probes through rolling circle DNA synthesis in situ, specifically reacted probes can be distinguished from nonspecifically bound probe molecules and other detection reagents. We modified an enzymatic target preparation procedure previously used for in situ amplification of padlock probes ${ }^{15}$ to produce single-stranded target strands with free $3^{\prime}$ ends downstream of the recognition sequences for the padlock probes, allowing target-primed amplification of circularized probes. Thus, the target strand can first act as the template for circularization of the probes and then prime RCA, thus avoiding topological inhibition of replication and ensuring efficient retention of the amplification products at the original location of the target sequences (Fig. 1). Any $3^{\prime}$ end sequence of the target strand that protrudes beyond the probe-recognition site can be removed by the $3^{\prime}-5^{\prime}$ exonucleolytic activity of the $\Phi 29$ polymerase $^{21}$, until the enzyme can use the padlock probe to template the RCA reaction. The RCA product, continuous with the target strand, consists of hundreds of single-stranded copies of the probe, repeated in tandem and available for detection by fluorophore-labeled hybridization probes directed towards tag sequences included in the non-targetcomplementary segment of the padlock probes $^{22}$.

\section{Mechanistic studies}

We evaluated the efficiency of the individual reactions steps of the integrated procedure in two separate series of experiments. First, the enzymatic target preparation procedure was investigated using different combinations of restriction enzymes and exonucleases to expose either the sense- or the antisense strand at the A3243G MELAS locus of the mitochondrial genome. The targets were 


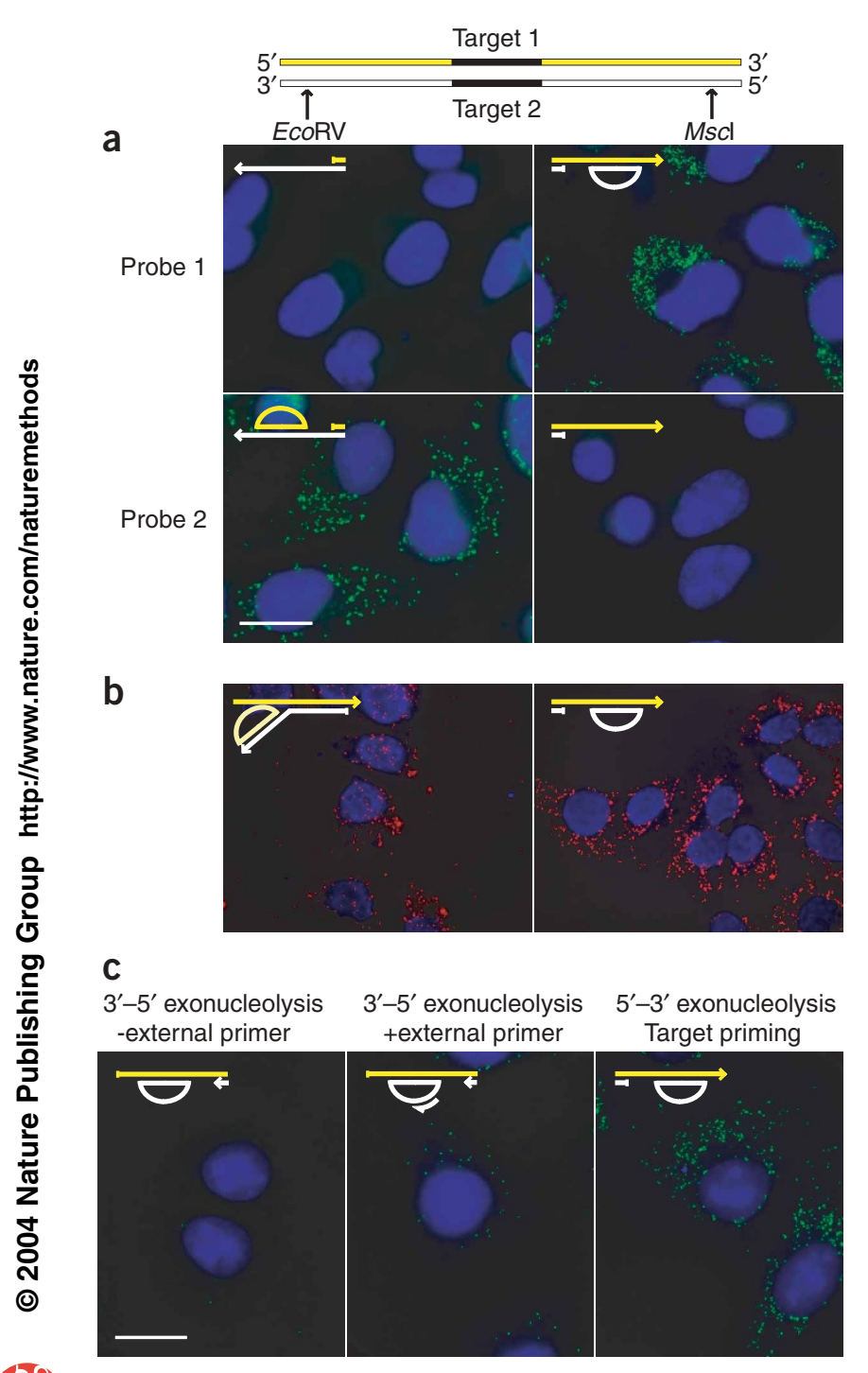

probed using the digoxigenin-labeled padlock probe ppMUT-DIG, which is specific for the sense strand, and these probes were then detected using fluorescent antibodies against digoxigenin (Fig. 2). This is in contrast to all subsequent experiments, in which unlabeled probes were rolling-circle amplified for enhanced detection. By using a direct labeling system, the target preparation method could be demonstrated independently of the target-primed RCA mechanism. Despite considerable nonspecific background, cytoplasmic signals indicating the presence of mitochondrial genomes were clearly more abundant under those two experimental conditions where the correct target strand was prepared for hybridization. The number of signals, and the nonspecific background, were similar when target sequences were prepared by standard chemical denaturation with $50 \%$ formamide in $2 \times$ SSC at $80^{\circ} \mathrm{C}$ for $90 \mathrm{~s}$ (data not shown). By contrast, combinations of enzymes that exposed the nontarget strand resulted in only nonspecific background signals (Fig. 2), similar to results obtained when no restriction or exonuclease digestion was undertaken (data not shown).

Next, we investigated under what conditions in situ-circularized probes could be amplified by an RCA procedure. For this purpose we used one padlock probe specific for the sense strand at the
Figure 3 | Target-primed RCA of padlock probes. Nuclei are shown in blue and RCA products in green or red. A schematic representation of the target- and restriction sites is shown on top of the images. Expected configurations of the target fragments are shown as insets. The sense strand is shown in yellow and the antisense strand in white; $5^{\prime}$ ends are indicated with bars and $3^{\prime}$ ends with arrowheads. Scale bars, $20 \mu \mathrm{m}$. (a) The two strand-specific padlock probes, 1 (ppMUTs; top row) and 2 (ppMUTas; bottom row), were amplified by RCA after target recognition. The mitochondrial genome in the M50 cell line was digested with either EcoRV (left) or MscI (right), making one or the other strand of the target locus single stranded after $\lambda$ exonuclease treatment. (b) Left, results from hybridization of a linear oligonucleotide probe to the target, with amplified detection through RCA of a preformed DNA circle. Right, results from target-primed RCA of the padlock probe ppWTs, detecting the same sequence as the linear probe. The target sequence in the W7 cell line was rendered single stranded by treatment with MscI and $\lambda$ exonuclease.

(c) The target strand for probe 1 was prepared single stranded with either a free $5^{\prime}$ or a free $3^{\prime}$ end. From left, the images show results from M50 cells displaying a free $5^{\prime}$ end of the target strand without and with added primer Lin33c, and from cells displaying a free $3^{\prime}$ end without added primer.

MELAS locus (ppMUTs) and one specific for the antisense strand (ppMUTas). Cells grown and fixed on slides were subjected to restriction digestion either $5^{\prime}$ or $3^{\prime}$ of the target sequence, followed by degradation of the $5^{\prime}$ ends using $\lambda$ exonuclease to expose the probe recognition sequences. This produced single-stranded sense or antisense target sequences with nearby free $3^{\prime}$ ends that could potentially prime RCA of reacted probes (Fig. 1). Signals were obtained for both the sense- and the antisense probes in samples treated to prepare the appropriate target sequences, and no signals were seen when padlock probes were combined with target sequences of the wrong polarity (Fig. 3a). The signals appeared as multiple brightly fluorescent spots with diameters of about $0.5 \mu \mathrm{m}$, probably representing individual rolling circle products, as sizes and signal intensities were similar to those of RCA products formed in solution and deposited on glass slides (data not shown). Larger signals of variable morphology may correspond to several adjacent rolling circle amplification products. The strength of the signals allowed a tenfold shorter camera exposure time to be used as compared to when padlock probes were detected with antibodies, and resulted in far greater contrast between signals and the background from autofluorescence.

We carried out experiments to compare the present method to previously published in situ RCA procedures ${ }^{12,15}$. The first strategy used preformed circular DNA strands to template RCA reactions in situ to detect the hybridization of sequencespecific linear probes to their target sequences ${ }^{12}$. To compare this strategy with ours, we used a 70 nucleotide (nt) linear probe (3243CMV), the $405^{\prime}$-most nucleotides of which were designed to hybridize to the wild-type variant at the MELAS locus, whereas the $30 \mathrm{nt}$ at the $3^{\prime}$ end were complementary to the padlock probe ppCMV93. The padlock probe was hybridized to the linear hybridization probe and converted to a circle by ligation. The linear probe and the circle were added to the slides and allowed to hybridize, and then washed as described in the original protocol ${ }^{12}$. The slides revealed cytoplasmic signals, but also abundant signals inside nuclei and outside cells, in contrast to the target-primed RCA experiment done in parallel where signals were confined to the cytoplasm (Fig. 3b). Similar results were obtained whether target DNA was rendered single stranded by enzyme treatment or by heat denaturation. 

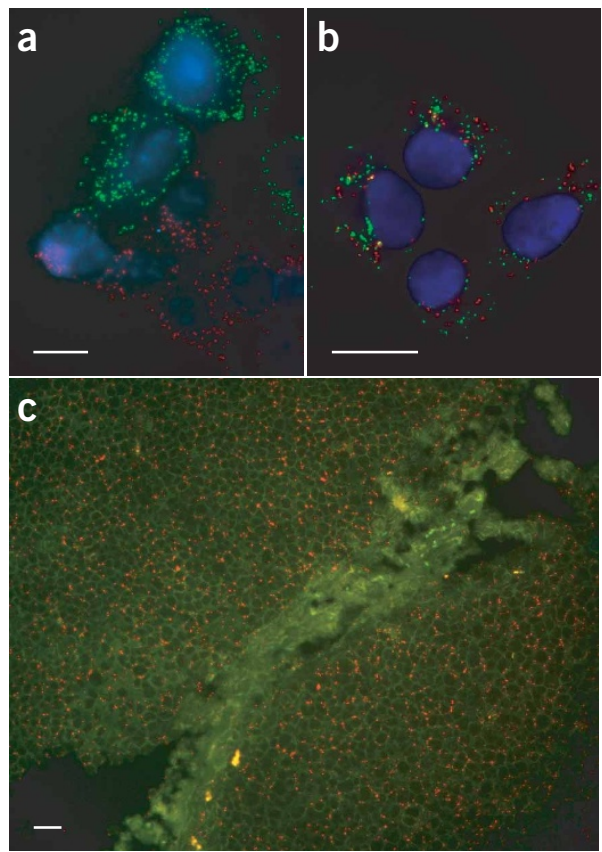

Next, we compared our procedure with a strategy where target sequences with nearby $5^{\prime}$ ends were obtained by enzyme treatment and padlock probes were amplified using an added oligonucleotide primer $^{15}$. In this comparison we added the ppMUTs probe to samples where restriction digested target sequences had been made single stranded using exonuclease III, producing a free $5^{\prime}$ end upstream of the probe recognition site. When no primer was added, almost no signals appeared, because no $3^{\prime}$ ends capable of priming RCA were created. When a primer complementary to the padlock probe was added to such reactions, about tenfold more signals were detected. The efficiency was still at least ten times lower, however, than when the same probe was used to detect target sequences prepared to have nearby free $3^{\prime}$ ends, allowing signals to be amplified by target-primed RCA (Fig. 3c). We used the $\Phi 29$ DNA polymerase to perform the RCA in these experiments. To reproduce the conditions of the original publication $^{15}$, we also carried out the RCA with ThermoSequenase over
Figure 4 | In situ genotyping of the mitochondrial A3243G point mutation. We applied the target-primed RCA method to detect two padlock probes, each specific for one of the two single-nucleotide variants of the target sequence. Signals from the two probes are shown in red (ppWTs) and green (ppMUTs) and the DAPI-stained cell nuclei are shown in blue. Scale bars, $20 \mu \mathrm{m}$. (a) Genotyping of the W20 and M50 cell lines, homoplasmic for the two different genotypes, in vitro cultured together on a slide. (b) Genotyping of the heteroplasmic G55-1.1 cell line. (c) Genotyping of mitochondrial genomes in a tonsil tissue section expected to contain only the wild-type target sequence. We subjected the tissue section to the same target-primed RCA procedure as the in vitro-cultured cell lines.

night, but this did not give rise to detectable signals in our hands (data not shown).

We further examined how the size of the target fragment affects the detection efficiency. By using target sequences located on different target fragments generated by the same restriction enzyme, we found that a 2,992 base pair (bp) restriction fragment yielded five times more signals than a $534 \mathrm{bp}$ fragment, probably reflecting loss of some of the shorter fragments after restriction digestion (see Supplementary Note and Supplementary Fig. 1 online). We also investigated the ability of the Ф29 DNA polymerase to degrade $3^{\prime}$ end sequences until they can serve as primers for the RCA reaction. When target sequences were prepared having free $3^{\prime}$ ends at different distances from the site of probe binding, we found that a $3^{\prime}$ end located $130 \mathrm{nt}$ downstream of the circularized probe was detected with half the efficiency compared to a target where the $3^{\prime}$ end was located immediately downstream of the probe-binding site (see Supplementary Note, Supplementary Fig. 2 and Supplementary Table 1).

\section{In situ genotyping of mitochondrial genomes}

We used a pair of padlock probes specific for the two sequence variants to genotype the mitochondrial A3243G point mutation in situ in three cell lines. Two cell lines that were homoplasmic for either mitochondrial sequence variant were cocultured, fixed on a glass slide and then subjected to target-primed RCA of in situcircularized probes. RCA products were detected by hybridization of two fluorescent oligonucleotide probes with sequences identical to distinct tag sequences in the two variant-specific padlock probes (Fig. 4a). The single-nucleotide difference between mitochondrial

Table 1 | Padlock probes and detection probes

\begin{tabular}{|c|c|}
\hline Probe & Sequence \\
\hline ppMUT-DIG & 5'-P-CTGCCATCTTAACAAACTTTCTCTTTATG(DIG)CTTAGTGACCTGCAG(DIG)TATTTCTCTTTCGATTACCGGGCC-3' \\
\hline ppWTs & 5'-P-CTGCCATCTTAACAATTCCTTTTACGACCTCAATGCTGCTGCTGTACTACTCTTCTGCGATTACCGGGCT-3' \\
\hline ppMUTs & 5'-P-CTGCCATCTTAACAACCTTTCCTACGACCTCAATGCACATGTTTGGCTCCTCTTCTGCGATTACCGGGCC-3' \\
\hline ppMUTas & 5'-P-GCCCGGTAATCGCCTTTCACGACCTCAATGCACATGTTTGGCTCCGCGACTATTITGTTAAGATGGCAGG-3' \\
\hline ppMSCas & 5'-P-CCTCATTGTACCCATTTTTCTACGACCTCAATGCTGCTGCTGTACTACTCTTCTATGCCAACCTCCTACT-3' \\
\hline ppMSCs & 5'-P-TAAGAAGAGGAATTGCCTTTCCTACGACCTCAATGCACATGTTTGGCTCCTCTTCCCATGGGTATGTTGT-3' \\
\hline ppCMV93 & 5'-P-СCTCCCATCATATTAAAGGCTTTCTCTATGTTAAGTGACCTACGACCTCAATGCTGCTGCTGTACTACTCTTCCTAAGGCATTCTGCAAACAT -3' \\
\hline 3243CMV & 5'-P-TATGCGATTACCGGGCTCTGCCATCTTAACAAACCCTGTTTAATATGATGGGAGGATGTTTGCAGAATGC-3' \\
\hline $\operatorname{Lin} 33 c$ & 5'-GGAGCCAAACATGTGCATTGAGG-3' \\
\hline Lin16 & 5'-СУ3-ССТСАATGCTGCTGCTGTACTAC-3' \\
\hline $\operatorname{Lin} 33$ & 5'-FITC-CCTCAATGCACATGTTTGGCTCC-3' \\
\hline
\end{tabular}

The padlock probes ppMUT-DIG and ppCMV93 were kindly provided by Eurogentec. The other padlock probes, the Lin33c primer and $3243 \mathrm{CMV}$ were purchased from DNA Technology A/S. The Lin16 and Lin33 fluorescence-labeled probes were purchased from Thermo Hybaid. P, $5^{\prime}$ phosphate; DIG, digoxigenin. Target-complementary sequences of padlock probes are shown in italics and tag sequence segments of padlock probes used for fluorescence detection are color coded. 
genomes in the two cell lines was clearly visualized, with one class of cells showing predominantly green signals and the other one red signals. The strong, discrete signals also allowed the distribution of the two mitochondrial genome variants to be studied within cells of a heteroplasmic cell line (Fig. 4b). By counting red and green fluorescence signals in 31 cells, we estimated the average heteroplasmy level as $66 \% \pm 8 \%$ (mean \pm s.d.) mutant mitochondrial DNA molecules in this cell line, in good agreement with the result of $64 \% \pm 7 \%$ obtained for the same cell line using gel-based quantification $^{23}$ (see Supplementary Fig. 3 and Supplementary Methods for details). We genotyped the same locus in a tonsillar tissue section, demonstrating that the fluorescence from individual amplification products stands out prominently from any autofluorescence in this tissue (Fig. 4c). Only wild-type signals were seen in the sample we investigated, which is expected considering the low frequency of the mutation in the general population ${ }^{24}$.

\section{DISCUSSION}

We introduce here a procedure for analyzing individual DNA molecules in situ by target-primed RCA of in situ-circularized padlock probes, with specificity sufficient to resolve singlenucleotide variation. This subtle target variation is converted into

윽 distinct tag sequences in the RCA products that are easily decoded by low-stringency hybridization of a standard pair of color-coded oligonucleotide detection probes. Because the localized signal amplification strictly requires a sequence-dependent padlock probe circularization, there is no background arising from nonspecifically bound probes and thus stringent or extensive washes are not required. Furthermore, samples are never exposed to temperatures over $37^{\circ} \mathrm{C}$ and they can be fixed by mild ethanol treatment, preserving cellular morphology.

We applied our method to study sequence variation in the mitochondrial genome, enabling analysis of the distribution of mitochondrial genomes within cells. The overall detection efficiency was estimated to be about $10 \%$ of all potential target sequences in the cells, based on the number of mitochondrial genomes in the cells as determined by real-time PCR (data not shown). We estimated the proportion of mutant and wild-type target sequences in individual heteroplasmic cells by counting individual amplification products, and the average result of this digital measurement was in good agreement with the result from standard PCR estimation at the population level. A detailed analysis of the intracellular distribution of mutant and wild-type mitochondrial genomes will be presented elsewhere (M.N., Karoly Szuhai, Hans Vrolijk, G.J., J. Antonie Maassen, A.K.R. et al., unpublished data). The present method allows, for the first time, robust analysis of the inter- and intracellular distribution of mitochondrial single-nucleotide DNA sequence variants. Our molecular approach for robust, specific analysis of single DNA molecules should also be applicable to localized detection of DNA sequences in other contexts, including DNA molecules located in the cytoplasm of cells, such as episomal viral or bacterial genomes, and in situ reverse-transcribed cDNA. The current detection efficiency of $10 \%$, however, precludes the use of the method for genotyping of nuclear single-copy genes in single cells.

Target-primed RCA of in situ-reacted padlock probes is a conceptually attractive procedure, combining highly specific detection with an essentially background-free amplification step. We compared this procedure experimentally to previously described procedures for DNA detection in situ. In our hands, amplification of preformed DNA circles ${ }^{12}$ resulted in unacceptably high background, precluding detection of low-copy-number target sequences (Fig. 3b). Padlock probes that form circles only upon target recognition provide greater specificity of detection. We found that RCA templated by padlock probes that remain bound to their target sequences is relatively inefficient if an external primer is used $^{15}$, probably because of steric hindrance ${ }^{16}$. As reported herein, we overcame this problem by arranging for the target molecule to serve as the amplification primer, which has the added benefit that the amplification products remain firmly anchored.

In conclusion, target-primed RCA of in situ-reacted padlock probes allows specific nucleic acid sequences to be investigated in cells and in tissue preparations. By identifying individual target molecules, digital information about their abundance and location can be obtained. The method permits studies of mitochondrial DNA organization that may provide fundamental insights into the pathogenesis of mitochondrial disease. It may also find applications in in situ studies of infectious organisms, and in the analysis of expressed members of conserved gene families, splice variants and allelic sequence variants, as well as arrays of samples, both in research and in routine pathological examinations.

\section{METHODS}

Sample pretreatments. The M50, W7, W20 and G55-1.1 cell lines ${ }^{23}$ were cultured and fixed on glass slides (Supplementary Methods). We prepared cells for in situ analyses by treatment with $0.01 \%$ pepsin (Sigma) in $0.1 \mathrm{M} \mathrm{HCl}$ for $90 \mathrm{~s}$ at $37{ }^{\circ} \mathrm{C}$, followed by brief washes in PBS. Anonymous tonsil tissue sections, fresh frozen and fixed in acetone, were obtained from the Department of Genetics and Pathology at the Uppsala University Hospital. Target-primed RCA of tissue samples was carried out as described for the cultured cells, except that the pepsin treatment was extended to five minutes.

We carried out the following reactions in a $50 \mu \mathrm{l}$ reaction volume under a $24 \times 55-\mathrm{mm}$ cover slip, or in reaction chambers defined by a silicone mask placed directly onto the slides, prepared essentially as previously described ${ }^{25}$ (for details see Supplementary Methods).

Enzymatic target preparation. We made target sequences accessible for hybridization by digestion with a combination of restriction and exonuclease enzymes ${ }^{26}$. The DNA was first restriction digested using $0.5 \mathrm{U} / \mu \mathrm{l}$ of $\mathrm{Msc}$ ( New England Biolabs) or EcoRV (AP Biosciences) at $37{ }^{\circ} \mathrm{C}$ for $30 \mathrm{~min}$ in the corresponding supplied buffers supplemented with $0.2 \mu \mathrm{g} / \mu \mathrm{l}$ BSA (New England Biolabs). We then rinsed the slides in buffer A $(0.1 \mathrm{M}$ Tris- $\mathrm{HCl}$ $\mathrm{pH} 7.5,0.15 \mathrm{M} \mathrm{NaCl}$ and $0.05 \%$ Tween-20). We made the ends of the restriction fragments single-stranded by exonucleolysis, using $0.2 \mathrm{U} / \mu \mathrm{l}$ of either the $3^{\prime}-5^{\prime}$ exonuclease III (New England Biolabs) or the $5^{\prime}-3^{\prime} \lambda$ exonuclease (New England Biolabs), at $37{ }^{\circ} \mathrm{C}$ for $15 \mathrm{~min}$ in the corresponding supplied buffers supplemented with $0.2 \mu \mathrm{g} / \mu \mathrm{l} \mathrm{BSA}$ and $10 \%$ glycerol. After incubation the slides were rinsed in buffer A.

Padlock probe hybridization and ligation. Oligonucleotide sequences are presented in Table 1. For direct detection of padlock probes, we hybridized $20 \mathrm{nM}$ of the padlock probe ppMUT-DIG in a solution of $2 \times$ SSC, $20 \%$ formamide and $0.5 \mu \mathrm{g} / \mu \mathrm{l}$ sonicated salmon sperm DNA at $37{ }^{\circ} \mathrm{C}$ for $15 \mathrm{~min}$. For RCA detection, we hybridized $100 \mathrm{nM}$ of padlock probe under the same conditions as 
for direct detection. We removed excess probe by a wash in buffer $\mathrm{B}$ $\left(2 \times\right.$ SSC, $0.05 \%$ Tween-20) for $5 \mathrm{~min}$ at $37{ }^{\circ} \mathrm{C}$ and then a rinse in buffer A. Padlock probes were circularized in $10 \mathrm{mM}$ Tris-acetate $\mathrm{pH}$ 7.5, $10 \mathrm{mM} \mathrm{MgAc} 2,250 \mathrm{mM} \mathrm{NaCl}, 1 \mathrm{mM}$ ATP, $0.2 \mu \mathrm{g} / \mu \mathrm{l} \mathrm{BSA}$ and $0.1 \mathrm{U} / \mu \mathrm{l}$ T4 DNA ligase (Amersham Biosciences) at $37{ }^{\circ} \mathrm{C}$ for $15 \mathrm{~min}$. If the padlock probes were not detected through RCA, we washed the slides stringently and visualized bound probe with two layers of fluorescence-labeled antibodies (Supplementary Methods). Slides for RCA detection were washed once in buffer B series of $70 \%, 85 \%$ and $100 \%$ ethanol.

Rolling circle amplification. ${ }^{14,16,27,28}$ We found that the use of rubber silicone reaction chambers greatly improved the efficiency of the RCA reaction. Therefore, we performed the RCA reactions on dehydrated slides in $50 \mathrm{mM}$ Tris- $\mathrm{HCl} \mathrm{pH} 7.5,10 \mathrm{mM} \mathrm{MgCl}_{2}$, $20 \mathrm{mM}\left(\mathrm{NH}_{4}\right)_{2} \mathrm{SO}_{4}, 0.2 \mu \mathrm{g} / \mu \mathrm{l} \mathrm{BSA}, 1 \mathrm{mM}$ DTT, $0.25 \mathrm{mM}$ dNTP, $10 \%$ glycerol and $1 \mathrm{U} / \mu \mathrm{l} \Phi 29$ DNA polymerase (Fermentas) at $37{ }^{\circ} \mathrm{C}$ for $30 \mathrm{~min}$ in the silicone mask reaction chambers placed on a shaking platform, except for the tonsillar tissue section, where the RCA was carried out under a coverslip. After polymerization, we removed the masks and rinsed the slides in buffer $\mathrm{A}$. We detected the single-stranded RCA products by hybridizing $250 \mathrm{nM}$ of Lin 16 and Lin33 fluorescence-labeled oligonucleotide probes in a solution of $2 \times$ SSC, $20 \%$ formamide and $0.5 \mu \mathrm{g} / \mu \mathrm{l}$ salmon sperm DNA for $15 \mathrm{~min}$ at $37^{\circ} \mathrm{C}$. After a brief rinse in buffer A, we finally dehydrated the slides, mounted them in Vectashield (Vector Laboratories) containing $10 \mathrm{ng} / \mathrm{ml}$ DAPI and stored them at $4{ }^{\circ} \mathrm{C}$. Experimental details of the comparisons with related methods are described in Supplementary Methods.

Image analysis. We acquired Images using an epifluorescence microscope (Axioplan II, Zeiss), equipped with a $100 \mathrm{~W}$ mercury lamp, a CCD camera (C4742-95, Hamamatsu) and a computercontrolled filter wheel with excitation and emission filters for visualization of DAPI, FITC and Cy3. A $100 \times$ objective (Plan-neofluar, Zeiss) was used for all images, except in Figure 4a $(63 \times$, Plan-neofluar) and Figure $4 \mathbf{c}(20 \times$, Fluar $)$, and images were captured using $0.2 \mathrm{~s}$ exposure time. We collected images using the Imstar software (Imstar) and thresholded them in Adobe Photoshop Elements 2.0 (Adobe Systems).

Note: Supplementary information is available on the Nature Methods website.

\section{ACKNOWLEDGMENTS}

We thank 0 . Söderberg for providing tissue sections and 0 . Ericsson and J. Jarvius for suggesting the use of the silicone masks as reaction chambers. Eurogentec kindly supplied padlock probes. The work was supported by the Wallenberg and the Borgström Foundations, the Foundation for Medical Research in Uppsala, The Swedish Doctors' Association, the European Union integrated project MolTools, and the Swedish Research Councils for Medicine and for Natural and Technological Sciences.

\section{COMPETING INTERESTS STATEMENT}

The authors declare competing financial interests; see the Nature Methods website for details.

\section{Received 9 July; accepted 20 0ctober 2004}

Published online at http://www.nature.com/naturemethods/

1. Antson, D.-0., Isaksson, A., Landegren, U. \& Nilsson, M. PCR-generated padlock probes detect single nucleotide variation in genomic DNA. Nucleic Acids Res. 28, e58 (2000).
2. Hardenbol, P. et al. Multiplexed genotyping with sequence-tagged molecular inversion probes. Nat. Biotechnol. 21, 673-678 (2003).

3. Baner, J. et al. Parallel gene analysis with allele-specific padlock probes and tag microarrays. Nucleic Acids Res. 31, e103 (2003).

4. Nilsson, M. et al. Padlock probes: Circularizing oligonucleotides for localized DNA detection. Science 265, 2085-2088 (1994).

5. Nilsson, M. et al. Padlock probes reveal single-nucleotide differences, parent of origin and in situ distribution of centromeric sequences in human chromosomes 13 and 21. Nat. Genet. 16, 252-255 (1997).

6. Antson, D.0., Mendel-Hartvig, M., Landegren, U. \& Nilsson, M. PCR-generated padlock probes distinguish homologous chromosomes through quantitative fluorescence analysis. Eur. J. Hum. Genet. 11, 357-363 (2003).

7. Koch, J., Kølvraa, S., Petersen, K., Gregersen, N. \& Bolund, L. Oligonucleotidepriming methods for the chromosome-specific labelling of alpha satellite DNA in situ. Chromosoma 98, 259-265 (1989).

8. Pellestor, F., Girardet, A., Andreo, B. \& Charlieu, J.-P. A polymorphic alpha satellite sequence specific for human chromosome 13 detected by oligonucleotide primed in situ labelling (PRINS). Hum. Genet. 94, 346-348 (1994).

9. Pellestor, F., Girardet, A., Genevieve, L., Andreo, B. \& Charlieu, J.P. Use of the primed in situ labelling (PRINS) technique for a rapid detection of chromosomes 13, 16, 18, 21, X and Y. Hum. Genet. 95, 12-17 (1995).

10. Koch, J., Hindkjaer, J., Kølvraa, S. \& Bolund, L. Construction of a panel of chromosome-specific oligonucleotide probes (PRINS-primers) useful for the identification of individual human chromosomes in situ. Cytogenet. Cell Genet. 71, 142-147 (1995).

11. Krejci, K. \& Koch, J. Improved detection and comparative sizing of human chromosomal telomeres in situ. Chromosoma 107, 198-203 (1998).

12. Zhong, X., Lizardi, P.M., Huang, X., Bray-Ward, P.L. \& Ward, D.C. Visualization of oligonucleotide probes and point mutations in interphase nuclei and DNA fibers using rolling circle DNA amplification. Proc. Natl. Acad. Sci. USA 98, 3940-3945 (2001).

13. Lindstrom, U.M. et al. Artificial human telomeres from DNA nanocircle templates. Proc. Natl. Acad. Sci. USA 99, 15953-15958 (2002).

14. Lizardi, P.M. et al. Mutation detection and single-molecule counting using isothermal rolling-circle amplification. Nat. Genet. 19, 225-232 (1998).

15. Christian, A.T. et al. Detection of DNA point mutations and mRNA expression levels by rolling circle amplification in individual cells. Proc. Natl. Acad. Sci. USA 98, 14238-14243 (2001).

16. Banér, J., Nilsson, M., Mendel-Hartvig, M. \& Landegren, U. Signal amplification of padlock probes by rolling circle replication. Nucleic Acids Res. 26, 5073-5078 (1998).

17. Kuhn, H., Demidov, V.V. \& Frank-Kamenetskii, M.D. Rolling-circle amplification under topological constraints. Nucleic Acids Res. 30, 574-580 (2002).

18. Wallace, D.C. Mitochondrial diseases in man and mouse. Science 283, 1482-1488 (1999).

19. Goto, Y., Nonaka, I. \& Horai, S. A mutation in the tRNA(Leu)(UUR) gene associated with the MELAS subgroup of mitochondrial encephalomyopathies. Nature 348, 651-653 (1990).

20. van den Ouweland, J.M. et al. Mutation in mitochondrial tRNA(Leu)(UUR) gene in a large pedigree with maternally transmitted type II diabetes mellitus and deafness. Nat. Genet. 1, 368-371 (1992).

21. de Vega, M., Lazaro, J.M., Salas, M. \& Blanco, L. Mutational analysis of phi29 DNA polymerase residues acting as ssDNA ligands for $3^{\prime}-5^{\prime}$ exonucleolysis. J. Mol. Biol. 279, 807-822 (1998).

22. Blab, G.A., Schmidt, T. \& Nilsson, M. Sensitive and homogenous detection of single rolling-circle replication products. Anal. Chem. 76, 495-498 (2004).

23. van den Ouweland, J.M.W., Maechler, P., Wollheim, C.B., Attardi, G. \& Maassen, J.A. Functional and morphological abnormalities of mitochondria harbouring the tRNA-Leu(UUR) mutation in mitochondrial DNA derived from patients with maternal inherited diabetes and deafness (MIDD) and progressive kidney disease. Diabetologia 42, 485-492 (1999).

24. Majamaa, K. et al. Epidemiology of A3243G, the mutation for mitochondrial encephalomyopathy, lactic acidosis, and strokelike episodes: prevalence of the mutation in an adult population. Am. J. Hum. Genet. 63, 447-454 (1998).

25. Pastinen, T. et al. A system for specific, high-throughput genotyping by allelespecific primer extension on microarrays. Genome Res. 10, 1031-1042 (2000).

26. van Dekken, H., Pinkel, D., Mullikin, J. \& Gray, J.W. Enzymatic production of single-stranded DNA as a target for fluorescence in situ hybridization. Chromosoma 97, 1-5 (1988).

27. Fire, A. \& Xu, S.-Q. Rolling replication of short DNA circles. Proc. Natl. Acad. Sci. USA 92, 4641-4645 (1995).

28. Liu, D., Daubendiek, S.L., Zillman, M.A., Ryan, K. \& Kool, E.T. Rolling circle DNA synthesis: small circular oligonucleotides as efficient templates for DNA polymerases. J. Am. Chem. Soc. 118, 1587-1594 (1996). 